\title{
Swelling and Contraction of Ferrocyanide-Containing Polyelectrolyte Multilayers upon Application of an Electric Potential
}

\author{
Dorothee Grieshaber, ${ }^{\dagger}$ Janos Vörös, ${ }^{\dagger}$ Tomaso Zambelli, ${ }^{\dagger}$ Vincent Ball, ${ }^{\ddagger}$, Pierre Schaaf,,$\perp$ \\ Jean-Claude Voegel, ${ }^{\ddagger}, 8$ and Fouzia Boulmedais*,ll'
}

\begin{abstract}
ETH Zurich, Laboratory of Biosensors and Bioelectronics, Institute for Biomedical Engineering, Gloriastrasse 35, CH-8092 Zurich, Switzerland, Institut National de la Santé et de la Recherche Médicale, Unité 595, 11 rue Humann, 67085 Strasbourg Cedex, France, Université Louis Pasteur, Faculté de Chirurgie Dentaire, 1 place de l'Hôpital, 67000 Strasbourg, France, Centre National de la Recherche Scientifique, UPR 22, Institut Charles Sadron, 23 rue du Loess, BP 84047, 67034 Strasbourg Cedex 2, France, and Université Louis Pasteur, ECPM, 25 rue Becquerel, 67087 Strasbourg Cedex 2, France
\end{abstract}

Received June 14, 2008. Revised Manuscript Received September 15, 2008

\begin{abstract}
We developed a new platform at the interface of polyelectrolyte multilayers (PEMs) and electroactive polymers (EAPs) by combining the easy buildup of PEM thin films and the deformation characteristics of the EAPs. The PEM films were made of poly(L-glutamic acid) (PGA) and poly(allylamine hydrochloride) (PAH). After $\left[\mathrm{Fe}(\mathrm{CN})_{6}\right]^{4-}$ ions (FCIV) were added, cyclic voltammetry (CV) was performed, resulting in a reversible expansion and contraction of the film. The shape change as well as the film buildup prior to the cycling were monitored in situ using the electrochemical quartz crystal microbalance with dissipation monitoring (EC-QCM-D). Electrochemical atomic force microscopy (EC-AFM) images confirmed the rapid shape deformation. The process takes place in an aqueous environment under mild conditions (maximum potential of $600 \mathrm{mV}$ and no $\mathrm{pH}$ change), which makes it a promising tool for biomedical applications. In addition, the electrochemically active films are produced using the layer-by-layer (LbL) method that is already established in biotechnology and biomaterials science; therefore, the presented approach can be readily adapted in these areas, bringing about a new possibility for the nanoscale dynamic control of coating thickness in various applications.
\end{abstract}

\section{Introduction}

The layer-by-layer ( $\mathrm{LbL}$ ) deposition of polyelectrolyte multilayers (PEMs) has been developed as a promising tool with a wide variety of application fields such as in optical devices and optoelectronics, ${ }^{1,2}$ surface treatment, ${ }^{3,4}$ membranes or selectivity, ${ }^{5,6}$ chemical or biological detection, ${ }^{7,8}$ biotechnology,,${ }^{9,10}$ and biomaterials. ${ }^{11,12}$ This assembly technique is a versatile and inexpensive approach to the formation of thin films via the alternating adsorption of positively and negatively charged species from aqueous solutions. The layers are held together mainly by

* Author to whom correspondence should be addressed. E-mail: boulmedais@ics.u-strasbg.fr. Phone: +33 3884141 60. Fax: +33 38841 4099.

ETH Zurich.

* Institut National de la Santé et de la Recherche Médicale.

${ }^{\S}$ Université Louis Pasteur, Faculté de Chirurgie Dentaire.

"Institut Charles Sadron.

${ }^{\perp}$ Université Louis Pasteur, ECPM.

(1) DeLongchamp, D. M.; Hammond, P. T. Chem. Mater. 2004, 16, 4799.

(2) Shim, B. S.; Tang, Z. Y.; Morabito, M. P.; Agarwal, A.; Hong, H. P.; Kotov, N. A. Chem. Mater. 2007, 19, 5467.

(3) Wu, Z. Z.; Walish, J.; Nolte, A.; Zhai, L.; Cohen, R. E.; Rubner, M. F. Adv. Mater. 2006, 18, 2699.

(4) Shchukin, D. G.; Zheludkevich, M.; Yasakau, K.; Lamaka, S.; Ferreira, M. G. S.; Mohwald, H. Adv. Mater. 2006, 18, 1672.

(5) Kotov, N. A.; Magonov, S.; Tropsha, E. Chem. Mater. 1998, 10, 886.

(6) Harris, J. J.; Stair, J. L.; Bruening, M. L. Chem. Mater. 2000, 12, 1941.

(7) Bucur, C. B.; Schlenoff, J. B. Anal. Chem. 2006, 78, 2360.

(8) Caruso, F.; Niikura, K.; Furlong, D. N.; Okahata, Y. Langmuir 1997, 13, 3422.

(9) Zelikin, A. N.; Quinn, J. F.; Caruso, F. Biomacromolecules 2006, 7, 27. (10) Jewell, C. M.; Fuchs, S. M.; Flessner, R. M.; Raines, R. T.; Lynn, D. M. Biomacromolecules 2007, 8, 857 .

(11) Thierry, B.; Kujawa, P.; Tkaczyk, C.; Winnik, F. M.; Bilodeau, L.; Tabrizian, M. J. Am. Chem. Soc. 2005, 127, 1626.

(12) Vautier, D.; Hemmerle, J.; Vodouhe, C.; Koenig, G.; Richert, L.; Picart, C.; Voegel, J. C.; Debry, C.; Chluba, J.; Ogier, J. Cell Motil. Cytoskel. 2003, 56, 147. electrostatic interactions and may contain a number of different functional groups. PEM films can also be obtained by interlayer hydrogen bonding. ${ }^{13}$

The influence of an electric potential on the buildup and the dissolution of PEM films are being studied more and more. Van Tassel and co-workers investigated the influence of an applied electric potential on the adsorption of charged polymers. ${ }^{14,15}$ Controlled electrodissolution of PEM films based on the application of an electric potential was reported in order to obtain the controlled release of a bioactive molecule. ${ }^{16}$ Electroactive thin films were designed by the insertion of electroactive components, such as Prussian blue, in PEM films. This electroactive coating has the ability to release a fraction of its content after the application of a voltage and then to restabilize upon its removal. ${ }^{17,18}$

There are two types of PEMs: linearly and exponentially growing films. Because of the diffusion within the film during adsorption, exponentially growing films are less structured than linearly growing ones. ${ }^{19}$ Such gel-like, nonstructured films facilitate the diffusion of ions, such as ferrocyanide (FCIV). Hubsch et al. found that in [poly(L-glutamic acid)] (PGA)-[poly(allylamine hydrochloride)] (PAH) multilayers FCIV diffuses

(13) Kharlampieva, E.; Sukhishvili, S. A. Langmuir 2003, 19, 1235.

(14) Ngankam, A. P.; Van Tassel, P. R. Langmuir 2005, 21, 5865.

(15) Ngankam, A. P.; Van Tassel, P. R. Proc. Natl. Acad. Sci. U.S.A. 2007, 104, 1140 .

(16) Boulmedais, F.; Tang, C. S.; Keller, B.; Voros, J. Adv. Funct. Mater. 2006, 16,63 .

(17) DeLongchamp, D. M.; Hammond, P. T. Adv. Funct. Mater. 2004, 14, 224.

(18) Wood, K. C.; Zacharia, N. S.; Schmidt, D. J.; Wrightman, S. N.; Andaya, B. J.; Hammond, P. T. Proc. Natl. Acad. Sci. U.S.A. 2008, 105, 2280.

(19) Picart, C.; Mutterer, J.; Richert, L.; Luo, Y.; Prestwich, G. D.; Schaaf, P.; Voegel, J. C.; Lavalle, P. Proc. Natl. Acad. Sci. U.S.A. 2002, 99, 12531. 
through the whole multilayer architecture. Once it is in the film, it is trapped and cannot diffuse out of the film. The authors estimated the FCIV concentration in the film to be about 500 $\mathrm{mM}$, thus 500 times higher than in the solution that was used. ${ }^{20}$ In addition, PEM films consisting of PAH, chemically modified with a redox-active Os complex, and glucose oxidase have been found to expand and contract upon application of a potential. ${ }^{21}$ Another film has been investigated by Schlenoff et al. They reported on a system with multilayers of polyelectrolytes containing redox-active sites where the oxidation/reduction induced a color change. ${ }^{22}$

On the other hand, electroactive polymers (EAPs) have the ability to change their shape. Besides other activation methods, electrical excitation is one of the most convenient methods of producing elastic deformation in polymers. Among them, there is the class of ionic polymer gels. They are activated by a chemical reaction, which is induced by changing from an acidic to an alkaline environment and vice versa. This reversible chemical reaction causes the gel first to swell and then to become dense again. ${ }^{23}$ Another system, consisting of layers of polyacrylamide hydrogels, was presented by Liu et al. Their hydrogels increase the thickness by $10 \%$ within 1 min upon application of $3 \mathrm{~V}$, which induces a $\mathrm{pH}$ change. However, this approach is not reversible in a closed system because the $\mathrm{pH}$ change that drives the shape change arises from the electrolysis of water, which leads to gas formation and therefore causes instabilities in the system. ${ }^{24}$ Schreyer et al. found that polyacrylonitrile gel fibers have the ability to elongate and contract. Because of this ability, they can potentially be used as linear actuators. To allow for electrical activation, platinum has been deposited on the fibers, or the fibers have been combined with graphite fibers. These modified gel fibers were fully contracted after $10 \mathrm{~min}$, and the contraction was found to be reversible over several cycles. However, this rate of contraction is still significantly slower than that of muscle tissue. ${ }^{25}$ Another group of EAPs are ionic EAPs (e.g., conductive polymers (CPs)). They show the ability to swell during redox cycling because of the insertion of ions. Subsequently, removal/expulsion of the ions results in shrinkage. The activation of this process requires voltages of $1-5 \mathrm{~V}$. In summary, despite their favorable characteristics, such as the ability to be easily formed in various shapes, their engineering properties, the endurance of many cycles of actuation, and the potential integration with sensors to produce smart actuators, such EAP materials, are not yet fully solved. The drawback of such materials is that for applications, such as artificial organs, the required voltages lie in the range from hundreds to thousands of volts. ${ }^{23}$

In this article, we compare the interaction of ferrocyanide with three different polyelectrolyte multilayer films: PGA/PAH, PSS/PLL, and a composite of the two. On the basis of the type of film, the application of an electric potential produces a different swelling effect induced by the presence of ferrocyanide. Finally, the composite film built up from PGA/PAH capped by a PSS/ PLL film inhibits the swelling and deswelling of the film. The presented work is situated at the interface between EAPs and PEM films. Our aim was to combine the shape deformation of the EAPs with the easy and inexpensive formation of polymeric PEMs under mild conditions. This means that neither a drastic

(20) Hubsch, E.; Fleith, G.; Fatisson, J.; Labbe, P.; Voegel, J. C.; Schaaf, P.; Ball, V. Langmuir 2005, 21, 3664.

(21) Forzani, E. S.; Perez, M. A.; Teijelo, M. L.; Calvo, E. J. Langmuir 2002, $18,9867$.

(22) Schlenoff, J. B.; Laurent, D.; Ly, H.; Stepp, J. Adv. Mater. 1998, 10, 347.

(23) Bar-Cohen, Y. Proc. Inst. Mech. Eng., Part G 2007, 221, 553.

(24) Liu, Z. S.; Calvert, P. Adv. Mater. 2000, 12, 288.

(25) Schreyer, H. B.; Gebhart, N.; Kim, K. J.; Shahinpoor, M. Biomacromolecules 2000, 1, 642 .
$\mathrm{pH}$ change nor a high applied voltage nor complicated chemical modifications are used. Furthermore, with regard to later applications, our system has shorter reaction times than previously developed materials. Because PEM films are held together via ionic bonds, we used the approach to change their shape through manipulating the electrostatic equilibrium by applying low voltages and thereby changing the oxidation state of ions in the film.

\section{Materials and Methods}

2.1. Materials. All measurements were carried out in a $10 \mathrm{mM}$ 4-(2-hydroxyethyl)piperazine-1-ethane-sulfonic acid (HEPES, Fluka Chemie GmbH, Switzerland) solution containing $100 \mathrm{mM} \mathrm{KCl}$. The $\mathrm{pH}$ of the buffer solution was adjusted to 7.4. The buffer was prepared with ultrapure water (Milli-Q gradient A 10 system, Millipore Corporation) and filtered $(0.2 \mu \mathrm{m})$ prior to use. For FTIR measurements, $\mathrm{D}_{2} \mathrm{O}$ (deuterium oxide $99 \% \mathrm{D}, \mathrm{AS}-\mathrm{A} 339$, Aldrich) was used instead of ultrapure water.

The polymers polyethyleneimine (PEI) (MW = $25000 \mathrm{~g} / \mathrm{mol}$, branched,408727), poly(L-glutamic acid)(PGA) (MW $=15000-50000$ $\mathrm{g} / \mathrm{mol}, \mathrm{P} 4761)$, poly(allylamine hydrochloride) $(\mathrm{PAH})(\mathrm{MW}=70000$ $\mathrm{g} / \mathrm{mol}, 28,322-3)$, poly (sodium 4-styrenesulfonate) $(\mathrm{PSS})(\mathrm{MW}=$ $70000 \mathrm{~g} / \mathrm{mol}, 24,305-1)$, and poly(L-lysine) (PLL) (MW = 15000 $\mathrm{g} / \mathrm{mol}$, P7890) were used to build up the PEMs. They were all purchased from Sigma, Switzerland. All polymers were used at a concentration of $1 \mathrm{mg} / \mathrm{mL}$ in HEPES buffer, which was sufficient to reach a saturating adsorption at a temperature of $25^{\circ} \mathrm{C}$.

Potassium ferrocyanide (FCIV, $1 \mathrm{mM}, \mathrm{K}_{4} \mathrm{Fe}(\mathrm{CN})_{6}$, Sigma, Switzerland) prepared in HEPES buffer was used for the electrochemical measurements.

Gold-coated sensor crystals (Q-Sense AB, Sweden) were used for the QCM-D measurements. The AFM measurements were carried out with indium tin oxide (ITO)-coated substrates (Microvacuum, Hungary).

2.2. Methods. Polyelectrolyte multilayer (PEM) films were built up by alternating the adsorption of positively and negatively charged polymers onto either a gold or an ITO surface. PEI was used as an initiator layer for all films regardless of their polymer combinations. Subsequently, up to 10 bilayers of PGA/PAH, PSS/PLL, or combinations of the two were adsorbed. Each polymer was injected for $5 \mathrm{~min}$. For QCM-D measurements, the polymers were adsorbed without a buffer rinse between steps. For AFM measurements, the excess was removed, and subsequently the system was rinsed with a pipet because of the lack of a flow cell. After extensive rinsing, following the last adsorption step, the film was exposed to FCIV for 10 min. A film constituted of a PEI layer and $n$ subsequent PGA/ PAH bilayers will be denoted by PEI-(PGA-PAH) ${ }_{n}$ and, after contact with FCIV solution, by PEI-(PGA-PAH) ${ }_{n}$-FCIV.

The quartz crystal microbalance with dissipation monitoring (QCM-D) (Q-Sense AB, Sweden) measures changes in the frequency $f$ and the dissipation factor $D$ of an oscillating quartz crystal upon adsorption of a viscoelastic layer. ${ }^{26-28}$ The quartz crystal is excited at its fundamental frequency $(5 \mathrm{MHz})$, and measurements are performed at the first, third, fifth, and seventh overtones, corresponding to 5, 15, 25, and $35 \mathrm{MHz}$. The QCM-D measurement is sensitive to the amount of water associated with the adsorbed molecules and senses the viscoelastic changes in the interfacial material. ${ }^{26}$ The dissipation changes, as well as frequency changes, allow for having a qualitative prediction of the changes in the mechanical properties of the film. Usually, an increase in dissipation is interpreted as a decrease in stiffness. In the Results and Discussion section, only the normalized negative frequency shifts of the third overtone are presented. The thickness of the adsorbed layers can be estimated using the viscoelastic Voigt model. For the evaluation, the

(26) Marx, K. Biomacromolecules 2002, 4, 1099.

(27) Hook, F.; Voros, J.; Rodahl, M.; Kurrat, R.; Boni, P.; Ramsden, J. J.; Textor, M.; Spencer, N. D.; Tengvall, P.; Gold, J.; Kasemo, B. Colloids Surf., B 2002, 24, 155. 1400. 

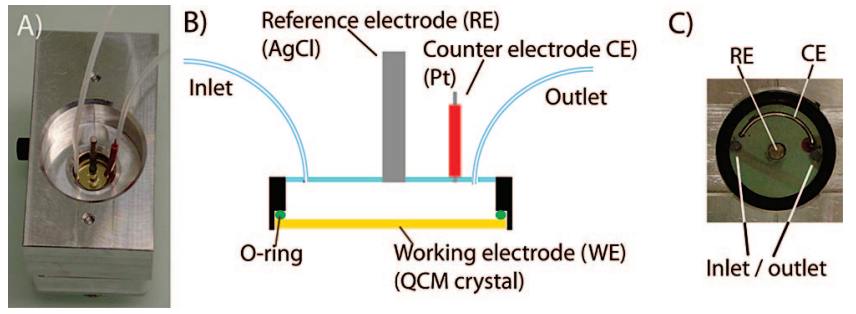

Figure 1. Electrochemical flow cell of the QCM-D. (A) Picture showing the arrangement of the electrodes and the transparent top. (B) Schematic cross-section of the flow cell. (C) Expanded view of the upper side of the flow cell. In the center of the transparent flow cell that is surrounded by an O-ring is the reference electrode (RE). The counter electrode (CE) is placed in a groove in the shape of a half-circle. Inlet and outlet tubes are on the left and right sides.

fluid density, fluid viscosity, and layer density are kept constant. The thickness is estimated using the third, fifth, and seventh overtones.

The QCM-D can also be combined with electrochemical sensing (EC-QCM-D) ${ }^{28,29}$ The flow cell used for our experiments is shown in Figure 1. Thereby, the gold coated QCM sensor acted as a working electrode. $\mathrm{An} \mathrm{Ag} / \mathrm{AgCl}$ wire on the upper side of the flow cell was used as the reference electrode and a platinum wire, placed in a small groove on the upper side of the flow cell, was used as a counter electrode. The top side was transparent and also contained inlet and outlet connections.

The film thickness of PEI-(PGA-PAH) $)_{5}$ and PEI-(PGA-PAH) 10 was measured in situ using a NanoWizard I BioAFM (JPK Instruments, Germany). The experiments in this article were carried out in tapping mode in the liquid state using an electrochemical cell to apply a potential during AFM scanning. ${ }^{30}$

Electrochemical (EC) measurements, such as cyclic voltammetry $(\mathrm{CV})$, were performed with a potentiostat in parallel with the abovementioned techniques. An IPS Jaissle PGU10V-1A-IMP-S potentiostat/galvanostat (Jaissle Elektronik GmbH, Germany) was used together with the QCM-D; an AMEL potentiostat/galvanostat (model 2053, AMEL electrochemistry, Italy) was used for EC-AFM experiments.

$\mathrm{CV}$ was performed prior to the measurements to condition the reference electrode. Therefore, a scan rate of $50 \mathrm{mV} / \mathrm{s}$ was used. After the deposition of the PEM film, an investigation of the multilayer properties was performed by applying $\mathrm{CV}$ using scan rates from 2 to $100 \mathrm{mV} / \mathrm{s}$. The scan range of the $\mathrm{CV}$ was $0-600 \mathrm{mV}$ versus $\mathrm{Ag} / \mathrm{AgCl}$. CV was applied prior to the injection of FCIV, whereas FCIV was in the film and in solution as well as after rinsing with FCIV only in the film.

Fourier transform infrared spectroscopy (FTIR) spectra were performed in attenuated total reflection (ATR) mode with an Equinox 55 (Bruker, France). The films were deposited on a trapezoidal ZnSe crystal (Graseby-Specac, Orpington, U.K.) located on the bottom of a flow cell (Graseby-Specac, Orpington, U.K.) by allowing each polyelectrolyte solution to circulate over the substrate for $5 \mathrm{~min}$. Two polyelectrolyte adsorption steps were separated by a buffer rinse for $10 \mathrm{~min}$. Polyelectrolyte and buffer circulation was allowed through Tygon tubing by means of a peristaltic pump. The infrared spectra of the film were acquired during each buffer rinse step in total attenuated reflection mode by accumulating 512 interferograms at a resolution of $2 \mathrm{~cm}^{-1}$. The signal transmitted through the $\mathrm{ZnSe}$ crystal was collected with a liquid-nitrogen-cooled mercury-cadmium telluride detector. During the deposition of the PEI-(PGA-PAH) multilayer film, the absorption spectrum was calculated as $-\log \left(T_{\text {layer }} /\right.$ $T_{\mathrm{PEI}}$ ), where $T_{\text {layer }}$ and $T_{\mathrm{PEI}}$ represent the transmission in the presence of the considered film and when PEI is adsorbed on the native $\mathrm{ZnSe}$ crystal, respectively. FCIV ions and PSS/PLL multilayers were adsorbed the same way. The spectra were acquired in the same way as for the deposition of the PEI-(PGA-PAH $)_{5}$ underlayer. The absorbance was then calculated by taking the transmission of the

(29) Dong, Y. G. Sens. Actuators, B 2005, 108, 622.

(30) Gewirth, A. A.; Niece, B. K. Chem. Rev. 1997, 97, 1129.
PEI-(PGA-PAH) 5 stratum as a reference in order to measure the change in ferrocyanide content (the cyanide groups give rise to a strong band centered at $2033 \mathrm{~cm}^{-1}$ ) as well as the deposition of PSS layers (giving rise to elongation peaks due to the sulfonate groups at 1035 and $1007 \mathrm{~cm}^{-1}$ ).

\section{Results and Discussion}

The main focus of this article is the swelling/deswelling of PGA/PAH films containing FCIV ions upon the application of an electric potential. First, the formation of the exponentially growing film is briefly documented. Then, different aspects of the swelling/deswelling phenomenon follow, describing when it is observed and to what extent the amplitude is obtained depending on the scan rate of the $\mathrm{CV}$ or the number of bilayers. The results obtained from the EC-QCM-D are compared to EC-AFM and FTIR measurements. The influence of PSS/PLL, a slightly exponentially growing $\mathrm{LbL}$ film, ${ }^{31}$ on the swelling/deswelling when it is adsorbed on top of the PGA/PAH film is studied. Even if (PGA-PAH $)_{n}$ and the (PSS-PLL $)_{n}$ were both of an exponential nature, we measured their refractive indices by means of optical waveguide lightmode spectroscopy and obtained values of 1.46906 and 1.48911, respectively. This suggests that the (PSS$\mathrm{PLL})_{n}$ films are more dense than (PGA-PAH) $)_{n}$ layers and hence should also be less permeable to FCIV anions. Thus, (PSS$\mathrm{PLL})_{n}$ layers appear to be good candidates for a capping layer. Finally, a mechanism is proposed on the basis of the different observations.

The deposition of PEI-(PGA-PAH) $)_{10}$ multilayer films was monitored in situ using the EC-QCM-D. Figure 2A represents the frequency shift and dissipation change directly obtained from the EC-QCM-D measurement. The frequency shift decreased after each adsorption step, which means that the adsorbed mass increased. PAH led to a high increase whereas the adsorption of PGA resulted in only a small increase in mass. All three curves - frequency shift, dissipation, and modeled thickness - show exponential film growth. This deposition is based on the "in" and "out" diffusion of PGA molecules through the entire film during each deposition cycle. ${ }^{32}$ By increasing the number of layers, the adsorption of PAH induced an increase in dissipation, whereas the subsequently adsorbed PGA led to a decrease. For 10 bilayers, the film was approximately $660 \mathrm{~nm}$ thick as shown in Figure 2B. Since the model was not able to give a reasonable approximation, the injection of the FCIV is not shown here. There was no difference observed with and without a buffer rinse in between the adsorption steps. That is why we did not use an additional rinsing step between the adsorption of the individual polymers (as described in the Methods section).

After the film deposition, a buffer solution containing FCIV ions was put into contact with the PEI-(PGA-PAH) ${ }_{10}$ film (last adsorption step in Figure 2A). The frequency shift increased whereas the dissipation decreased, which could indicate either a loss of material or a contraction of the film and less flexibility. To determine which reaction occurs, we performed the same experiment by FTIR spectroscopy in ATR mode. The spectra of PEI-(PGA-PAH) 5 after the adsorption of each PGA/PAH bilayer and after the uptake of FCIV ions and subsequent rinsing are shown in Figure 3. At each adsorption step of the PGA/PAH bilayers, the absorbance peaks of PGA (at 1635 and $1560 \mathrm{~cm}^{-1}$, corresponding respectively to amide $\mathrm{I}$ and $\mathrm{COO}^{-}$peaks) progressively increased. It appears that the absorbance increases in a nonexponential manner and even seems to level off after

(31) Tezcaner, A.; Hicks, D.; Boulmedais, F.; Sahel, J.; Schaaf, P.; Voegel, J. C.; Lavalle, P. Biomacromolecules 2007, 7, 86.

(32) Boulmedais, F.; Ball, V.; Schwinte, P.; Frisch, B.; Schaaf, P.; Voegel, J. C. Langmuir 2003, 19, 440. 
A)

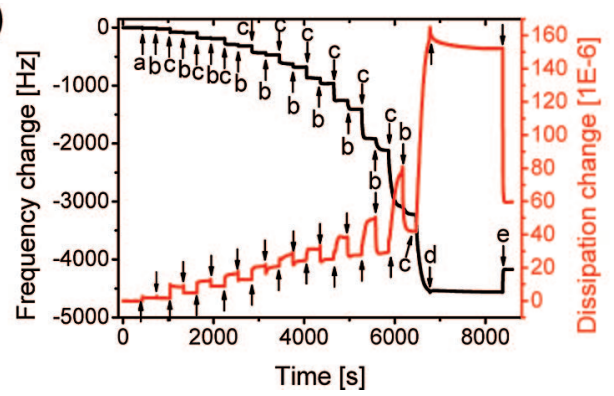

B)

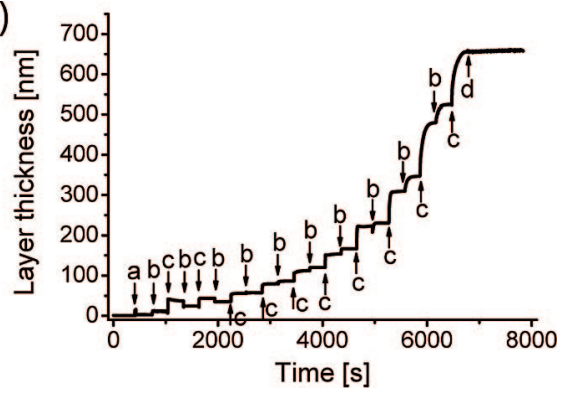

Figure 2. (A) Adsorption of PEI-(PGA-PAH) 10 -FCIV. The frequency shift decreased with increasing mass, whereas the dissipation increased. The last step represents the adsorption of FCIV. The arrows indicate the adsorption of the polymer layers and the FCIV. Those pointing to the frequency curve are labeled as a (PEI), b (PGA), c (PAH), d (buffer), and e (FCIV). (B) Modeled thickness of the adsorbed PEM film using the viscoelastic Voigt model. The arrows indicate the injection according to A.
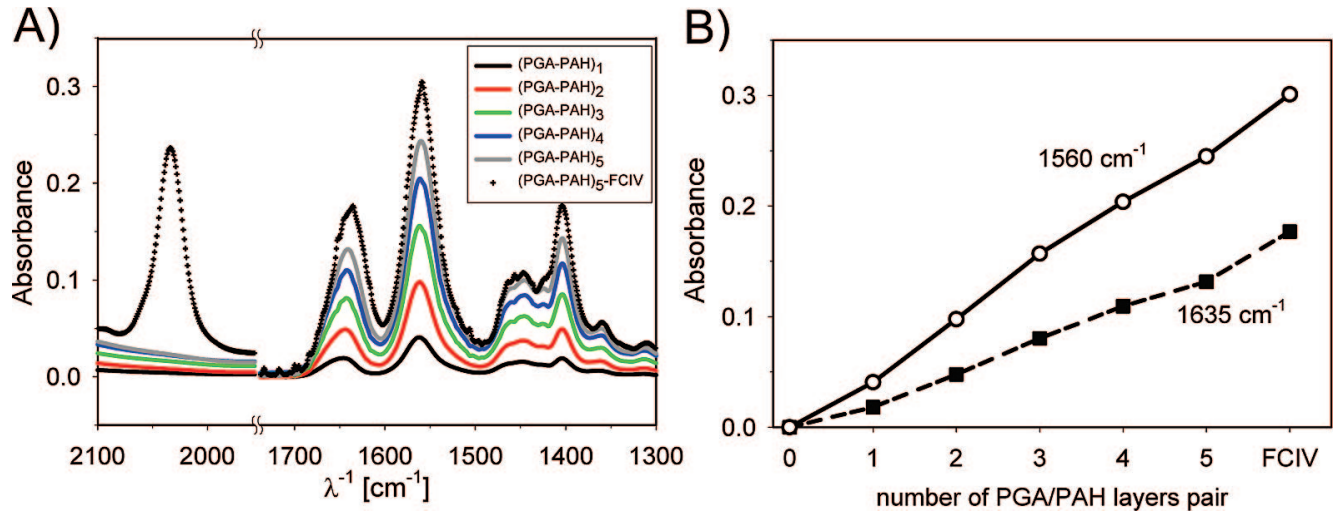

Figure 3. (A) Absorbance spectra of a PEI-(PGA-PAH) 5 -FCIV film obtained by FTIR in ATR mode. The inset displays the color of the spectra corresponding to the deposition of the indicated pairs of polyelectrolytes and the loading with FCIV. The peak at $2033 \mathrm{~cm}^{-1}$ is attributed to FCIV. (B) Evolution of the absorbance at $1560 \mathrm{~cm}^{-1}$ (peak due to the $\mathrm{COO}^{-}$groups of PGA) and at $1635 \mathrm{~cm}^{-1}$ (maximum in the amide I band of PGA) as a function of the number of PGA/PAH bilayer depositions and after loading with FCIV.

four to five layer pairs. In FTIR spectroscopy in the ATR mode, the film that is deposited on the substrate ( $\mathrm{ZnSe}$ crystal) is sensed by an evanescent wave with a penetration depth in the range of $1.5 \mu \mathrm{m}$. The light intensity is a function of the separation distance from the $\mathrm{ZnSe} /$ solution interface $(z)$. In the case where $z_{2}>z_{1}$, a PGA molecule located at a distance $z_{2}$ contributes less to the light absorption than does a molecule located at a distance $z_{1}$. The exponential increase in the number of deposited PGA molecules, combined with the exponential decrease in the intensity of light versus $z$, contributes to a measured linear increase of the absorbance for up to 8 layer pairs (data not shown). When the PGA/PAH film was put into contact with FCIV containing buffer, the FCIV peak at $2033 \mathrm{~cm}^{-1}$ appeared. This means that there was an uptake of FCIV by the film. At the same time, an increase in the intensity of PGA peaks was observed. This increase can be interpreted only as an increase in the PGA concentration in the region close to the $\mathrm{ZnSe}$ crystal. This strongly suggests that the film undergoes contraction with almost no film erosion upon FCIV loading. There is no indication of loss of material during FCIV adsorption. Therefore, in combination with EC-QCM-D measurements, the results suggest that the addition of FCIV ions contracts the film and renders it more rigid.

While the FCIV is in contact with the (PGA/PAH) $)_{10}$ film, CV measurements were performed at different scan rates and monitored by EC-QCM-D to follow the frequency shift and the dissipation change (Figure 4). The frequency shift decreased when the potential was increased from 0 to $600 \mathrm{mV}$, which means that the film expanded. Synchronically, the dissipation increased, which indicates a less rigid film. The film reversibly contracted to its original thickness and rigidity when the potential was decreased back to $0 \mathrm{mV}$. Subsequently, CVs from 0 to 600

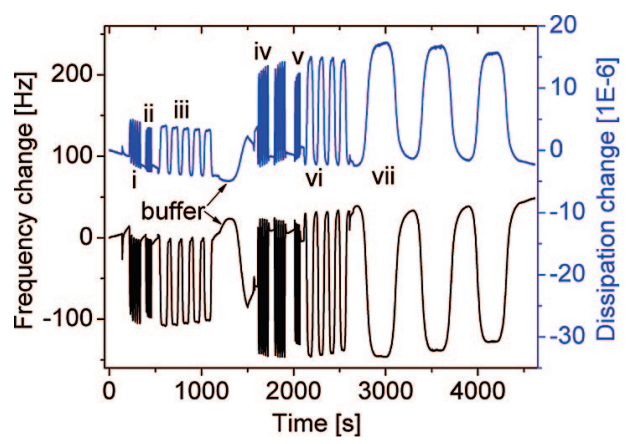

Figure 4. Frequency shift and dissipation change as a function of time on the PEI-(PGA-PAH) ${ }_{10}$-FCIV film. First, CV measurements from 0 to $600 \mathrm{mV}$ were made after adding FCIV (FCIV in the film and in solution) at scan rates of (i) 50, (ii) 100 , and (iii) $10 \mathrm{mV} / \mathrm{s}$. After the buffer rinse, the $\mathrm{CV}$ measurements were repeated at scan rates of (iv) 50, (v) 100, (vi) 10 , and (vii) $2 \mathrm{mV} / \mathrm{s}$.

$\mathrm{mV}$ were applied at different scan rates ranging from 2 to 100 $\mathrm{mV} / \mathrm{s}$ as shown in Figure $4 \mathrm{i}-$ iii. Independent of the scan rate, the frequency shift and dissipation change followed the potential change.

The swelling/deswelling phenomenon of PGA/PAH films in contact with FCIV ions under CV were found to be reversible. The fact that even at high scan rates the same expansion and contraction occurred indicates that the film reacts instantly. Control runs of CV on PEI-(PGA-PAH) $)_{10}$ before the injection of FCIV ion solution were also performed. Only negligible effects on the dissipation $(<1 \mathrm{E}-8)$ and on the frequency shift (around $0.5 \mathrm{~Hz}$ ) were observed. After being in contact with FCIV containing buffer, the PGA/PAH film was rinsed with buffer to 

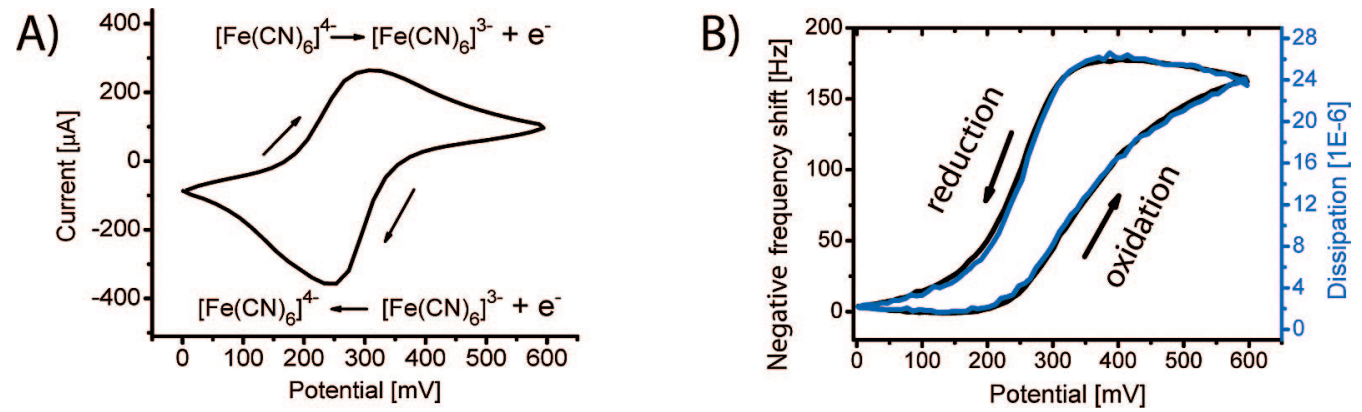

Figure 5. (A) Voltammogram showing oxidation and reduction peaks of a PEI-(PGA-PAH) 10 -FCIV film (FCIV only in the film). (B) Frequency shift and dissipation as a function of the applied potential during CV (scan rate $50 \mathrm{mV} / \mathrm{s}$ ) of the same film. Please note that the left $y$ axis shows the negative values of the frequency shift. This allows a visualization of the frequency and dissipation changes occurring simultaneously.
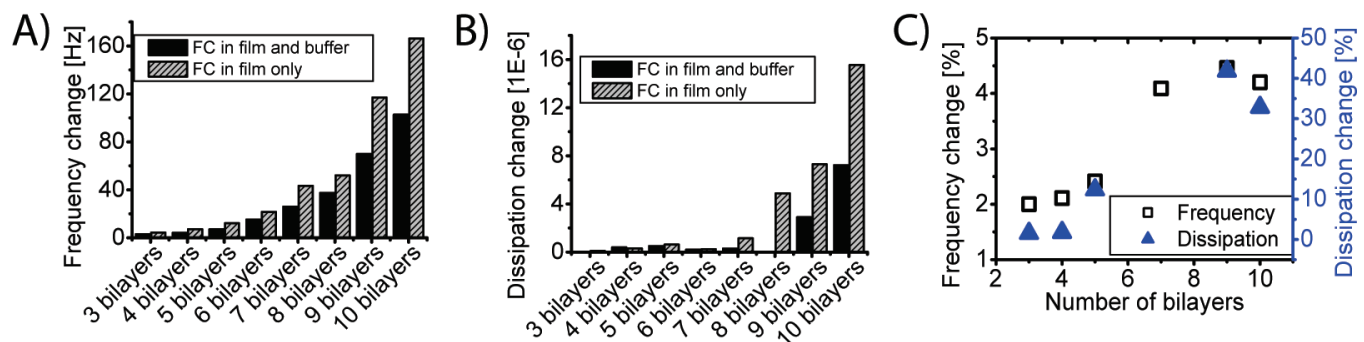

Figure 6. Absolute and percentage changes in the frequency shift and dissipation of PEI-(PGA-PAH) ${ }_{n}$-FCIV films with $3-10$ bilayers upon application of $\mathrm{CV}$ from 0 to $600 \mathrm{mV}$ at a scan rate of $50 \mathrm{mV} / \mathrm{s}$. (A) Change in the frequency shift during $\mathrm{CV}$ as a function of the number of layers when a positive potential was applied. (B) Corresponding dissipation changes. (C) Frequency shift and dissipation change as percentages of the values for the total film at $0 \mathrm{mV}$.
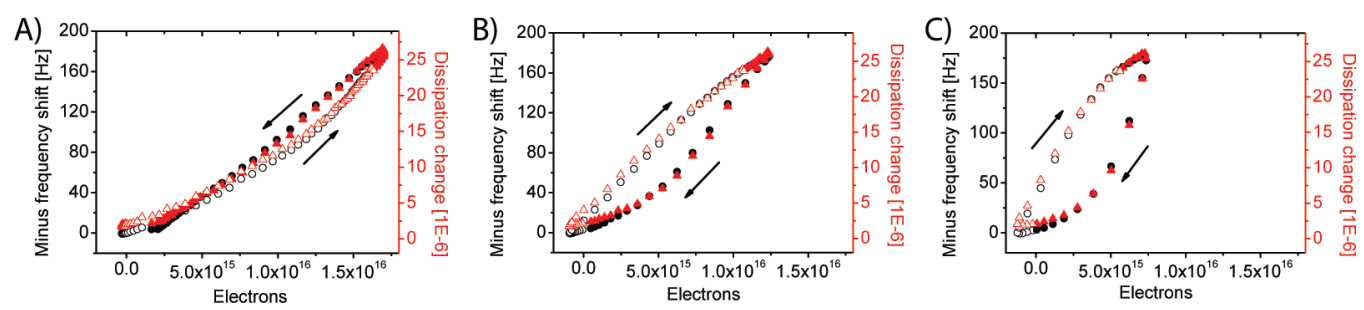

Figure 7. Absolute value of the frequency shift (o) and dissipation change $(\Delta)$ of PEI-(PGA-PAH) 10 -FCIV as a function of transferred electrons during CV from 0 to $100 \mathrm{mV}$. Different scan rates were applied: (A) 10, (B) 50, and (C) $100 \mathrm{mV} / \mathrm{s}$. The increase and decrease were nearly linear. In the case of faster scan rates, the contraction was faster than the swelling. Note that the left $y$ axes show the negative values of the frequency shift.

remove the FCIV ions remaining in solution. Then, CV measurements were performed with the same scan rates as before. Without FCIV ions in the supernatant, the amplitudes of the frequency shift and dissipation during $\mathrm{CV}$ were found to be even higher (Figure 4iv-vii). This can be explained by the presence of different counterions (chloride and FCIV ions) diffusing into the film upon oxidation of the FCIV ions. When FCIV ions are still in the supernatant, they will diffuse into the film and thereby reduce the cycling effect. However, without FCIV in the supernatant only chloride ions can diffuse into the film to maintain charge electroneutrality. As for the measurements with FCIV in solution, the thickness expansion did not significantly depend on the scan rate when the FCIV in solution had been removed. In addition, Figure 4 shows that five successive oxidation/reduction cycles can be performed without changing the performance of the device.

The cyclovoltammogram (current versus electric potential) monitored for a PEI-(PGA-PAH) 10 film after the adsorption of FCIV ions is shown in Figure 5A. During the increase in the potential from 0 to $600 \mathrm{mV}$ (scan rate $50 \mathrm{mV} / \mathrm{s}$ ), FCIV ions were oxidized to FCIII $\left(\left[\mathrm{Fe}(\mathrm{CN})_{6}\right]^{4-} \rightarrow\left[\mathrm{Fe}(\mathrm{CN})_{6}\right]^{3-}+\mathrm{e}^{-}\right)$. This reaction resulted in an oxidation peak of almost $300 \mu \mathrm{A}$. Because FCIII ions have one less charge than FCIV ions, the charge distribution in the film is disturbed. When the potential was decreased to $0 \mathrm{mV}$, FCIII ions were reduced to FCIV ions by gaining an electron, leading to a reductive peak current. For the same film, Figure 5B represents the absolute values of the frequency shift and dissipation as a function of the applied potential. This Figure shows the congruency of the two curves. The expansion and contraction of PGA/PAH films took place during the oxidation/reduction of the FCIV/FCIII ions with a maximum swelling/deswelling at the oxidation and reduction potentials of the FCIV ions. As described later (Figure 7), this indicates that the expansion and contraction are directly related to the number of transferred electrons.

The swelling/deswelling effect was studied for 3-10 bilayers of PGA/PAH. The frequency shift and dissipation amplitudes were measured during $\mathrm{CV}$ while FCIV ions were in solution and after the rinsing step. Figure 6A,B represents the frequency and dissipation shifts during $\mathrm{CV}$ with a scan rate of $50 \mathrm{mV} / \mathrm{s}$. For the investigated films, the thickness increases more after removing free FCIV ions in solution. Once these ions had been removed, no more changes in the swelling/deswelling behavior were detected, even after repeated rinsing steps.

Upon application of a potential, the frequency shift increases exponentially with the number of layers of the film as did the 
thickness of the film itself. The dissipation change is almost negligible for thin films and increases exponentially above seven bilayers. The amplitudes of changes in frequency and dissipation were also determined. In Figure 6, their changes are plotted as percentages of the total film frequency shift and dissipation change. By increasing the number of bilayers from 3 to 10 , the frequency shift and dissipation increase, respectively, from 2 to around $4 \%$ and from 0 to about $30-40 \%$ with respect to the frequency shift and the dissipation measured before oxidation. Additionally, CV measurements with PGA as the last layer were performed. After the adsorption of FCIV ions and subsequent rinsing, the swelling/deswelling effect of PEI-(PGA-PAH) ${ }_{10^{-}}$ PGA was significantly lower than for PEI-(PGA-PAH) ${ }_{10}, 1.3 \%$ instead of $4 \%$ in frequency shift and $15 \%$ instead of $32 \%$ in dissipation change (data not shown). PGA as a last layer therefore dramatically decreased the swelling/deswelling effect. Recently, by means of electrochemistry, Hubsch et al. studied the diffusion kinetics of FCIV ions into PGA/PAH films (with PGA or PAH as a last layer) when the films are brought into contact with a FCIV solution $(1 \mathrm{mM})$. When a PGA terminal film was compared to a PAH terminal film, the peak currents were found to be lower, but the areas of the oxidation peak were found to be equal for a contact time of $100 \mathrm{~min}$. Then the number of FCIV ions is only $20 \%$ less in PGA-ending films compared to PAH-ending films. ${ }^{20}$ In our case, the film is in contact with the FCIV solution for only $10 \mathrm{~min}$. The amount of FCIV is dramatically different (about 80\% fewer FCIV ions in PGA-terminal films compared to the number in PAH-terminal films). The areas of the oxidation peaks are clearly different for the two films. On the basis of electrochemical measurements, Takita et al. also reported that PGA-terminal PGA/PAH films take up fewer ferricyanide ions (FCIII), with a contact time of $15 \mathrm{~min} .{ }^{33}$ This effect was attributed to electrostatic repulsion between the negatively charged probe and the ending PGA or to the fact that a PGA-ending film is richer in PGA owing to PGA diffusion into the whole film thickness. Thus, less negatively charged FCIV ions can penetrate into a PGA-saturated film. Subsequently, these fewer ions induce a lower current during CV and, as we have shown for the first time here, a smaller swelling/deswelling effect.

We were also interested in knowing the number of FCIV ions that need to be oxidized to induce a change in the film thickness. By integrating the CV curves, we calculated the number of electrons transferred. Because in the absence of FCIV ions there was no oxidation/reduction current detected from the film only, the number of transferred electrons is identical to the number of FCIV ions that have been oxidized. Examples of experiments performed at different scan rates are given in Figure 7. Figure 7A depicts the changes in frequency shift and dissipation as a function of the number of transferred electrons at a scan rate of $10 \mathrm{mV} / \mathrm{s}$. When the number of electrons transferred increases through the increase in the applied potential, the absolute value of the frequency shift and the dissipation change also increase. When the number of electrons decreases, the frequency shift and the dissipation change decrease. Moreover, the frequency shift and the dissipation change are clearly superimposed, indicating that the swelling is proportional to the number of electrons transferred at $10 \mathrm{mV} / \mathrm{s}$. Figure 7B,C shows the evolution of the frequency shift and the dissipation change for scan rates of 50 and $100 \mathrm{mV} / \mathrm{s}$, respectively. For slow scan rates, the swelling and the deswelling were found to be linear functions of the number of transferred electrons. In the case of higher scan rates, especially for $100 \mathrm{mV} / \mathrm{s}$, the deswelling was not linear but was faster than the reduction of the FCIII ions. The absolute values of the initial

(33) Takita, R.; Yoshida, K.; Anzai, J. Sens. Actuators. B 2007, 121, 54. slopes of oxidation and reduction curves, obtained from Figure 7 increase as the scan rate increases. Concerning the frequency shift, the absolute values of the slopes are $0.5 \times 10^{-14}, 1.5 \times$ $10^{-14}$, and $3.0 \times 10^{-14} \mathrm{~Hz} / \mathrm{e}^{-}$for oxidation curves and $1.1 \times$ $10^{-14}, 1.7 \times 10^{-14}$, and $5.6 \times 10^{-14} \mathrm{~Hz} / \mathrm{e}^{-}$for reduction curves at scan rates of 10,50 , and $100 \mathrm{mV} / \mathrm{s}$, respectively. Furthermore, it needs to be mentioned that at higher scan rates the total number of transferred electrons was lower (i.e., at $100 \mathrm{mV} / \mathrm{s}$ only half of the number of electrons were transferred compared to the number transferred at $10 \mathrm{mV} / \mathrm{s}$ ). This decrease in current at higher scan rates indicated that the diffusion is rate-limiting (i.e., the currents also might be a contribution of the capacitive current). In case of slow scan rates, the film had enough time to more or less follow the oxidation and the reduction in equilibrium. At higher scan rates, where fewer electrons were flowing in total, the contraction took place faster than the expansion. The reason therefore might be that in the oxidized state the film did not have enough time to reach equilibrium. Upon FCIV oxidation, negatively charged counterions have to penetrate into the film to maintain charge electroneutrality. However, both of the monovalent counterions (FCIII and especially $\mathrm{Cl}^{-}$) have reduced ion bridge forming capability compared to that of the FCIV ions; therefore, the film expands. However, the breaking of existing ion bridges in the polymer film during oxidation might take longer than the reformation during reduction. This can be an explanation for the asymmetric expansion curves at high scan rates. This means that the ions were oxidized and contributed to the swelling but once the potential was decreased they were immediately reduced and therefore the film contracted faster. Furthermore, because the layer thickness changes during the CV scan, this provides a moving boundary for the reservoir from which the FCIV ions diffuse to the electrode. This could explain why the swelling is slower than the contraction at higher scan rates (i.e., the ions have to move less when the thickness is smaller).

To further verify the results from the EC-QCM-D, the thickness of the PEI-(PGA-PAH) 10$_{10}$-FCIV film was monitored by AFM in the liquid state before, during, and after the application of 600 $\mathrm{mV}$ (film deposition in Supporting Information). To obtain its thickness, the film was scratched, and the height difference between the ITO substrate and the film surface was evaluated. The film surface with a scratch is shown in Figure 8A. Next to the scratch there was a bulge originating from the material in the scratch. Further away, the film was flat and about $400 \mathrm{~nm}$ thick. The slightly smaller thickness obtained by the AFM (i.e., $\sim 400$ $\mathrm{nm}$ ) in comparison to the QCM-D (i.e., $\sim 650 \mathrm{~nm}$ ) might be attributed to the difference between the two techniques.

Figure $8 \mathrm{~B}$ shows the cross-section of a PEI-(PGA-PAH) $10^{-}$ FCIV film at 0 and $600 \mathrm{mV}$. The application of a potential of $600 \mathrm{mV}$ induced an $\sim 5-10 \%$ swelling of PGA/PAH films compared to the thickness at $0 \mathrm{mV}$. Lowering the potential back to $0 \mathrm{mV}$ reversibly decreased the film thickness to its original state. In the inset, a cross-section over several cycles (potential on/off) is shown. To obtain this graph, the AFM program took the surface of the film as the reference and imaged changes in the depth of the scratch. Therefore, the spikes in the profile are related to some impurities in the scratch (from film and/or solution) and do not represent an uneven film surface.

One possible explanation for the swelling/deswelling effect could be that the FCIII ions, obtained by the oxidation of FCIV, diffuse out of the film when a potential is applied. To test this hypothesis, we performed another experiment where a flow of buffer was applied during the electrochemical measurements. After the deposition of a PEI-(PGA-PAH) ${ }_{10}$ film, it was put into 
A)

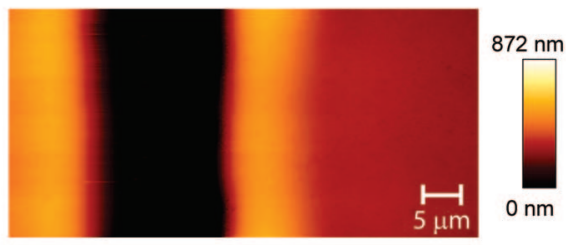

B)

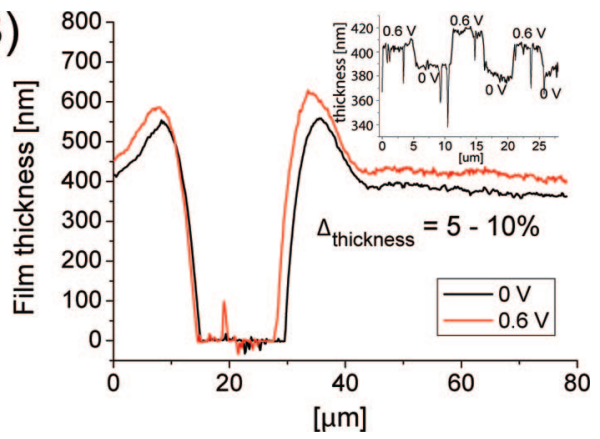

Figure 8. (A) AFM image of PEI-(PGA-PAH) 10 -FCIV with a scratch obtained by tapping mode in the liquid state to measure the film thickness. (B) Cross-section of the PEI-(PGA-PAH) 10 -FCIV film. A potential of $600 \mathrm{mV}$ induced a thickness increase of about 5-10\%. In the inset, the thickness change is monitored during the scan where the potential was repeatedly turned on and off.

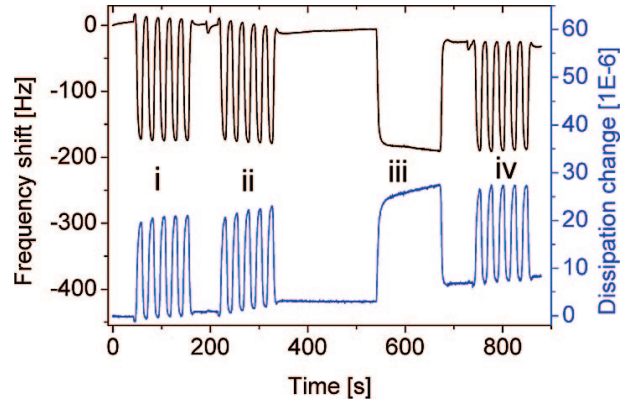

Figure 9. Frequency shift and dissipation as a function of time during (i) CV of a PEI-(PGA-PAH) 10 -FCIV film without the flow of buffer, (ii) with the flow of buffer, (iii) with a constant potential of $600 \mathrm{mV}$ for 2 min, and (iv) CV without flow.

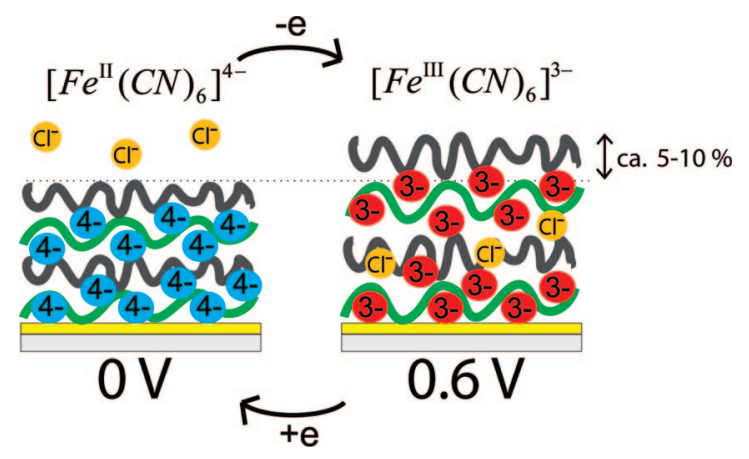

Figure 10. Schematics of a PGA/PAH multilayer with incorporated FCIV ions. When the FCIV ions are oxidized by the application of an electric potential, chloride ions enter the multilayer, and the film swells. When the FCIII ions are reduced to FCIV ions, chloride ions go out from the multilayer, and the film contracts.

contact with buffer containing FCIV ions. After the first rinsing step, CV $(50 \mathrm{mV} / \mathrm{s})$ was applied with and without the flow of buffer (Figure 9i,ii). Then, a constant potential of $600 \mathrm{mV}$ was applied for 2 min under flow (as shown in Figure 9iii). During this time, the supernatant was exchanged about four times. No significant decrease in the swelling/deswelling behavior was observed with the buffer flow whereas the Faradic current is slightly higher (about 5\%). This indicates that the oxidized FCIII ions remain in the film when a potential is applied. Otherwise, if the swelling had been induced by the release of FCIII ions into the buffer then the concentration of these ions would have decreased significantly as soon as the supernatant was replaced and the swelling would have decreased. Subsequent CV without flow supported these findings by showing almost the same magnitude of swelling (Figure 9iv).

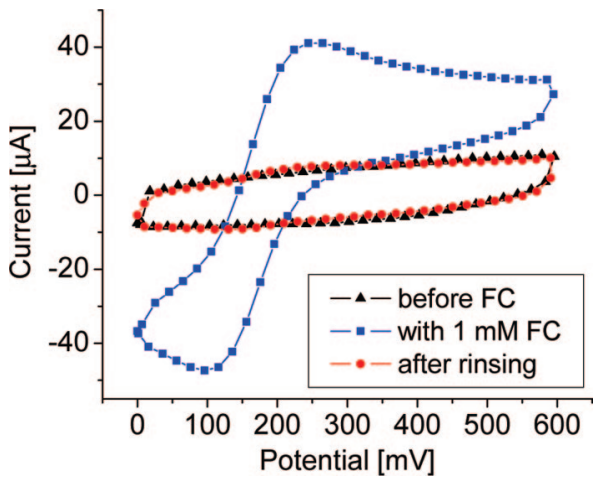

Figure 11. Voltammogram (50 mV/s) of PEI-(PSS-PLL) 5 film before, during. and after contact with FCIV solution.

Our proposed mechanism of the swelling/deswelling phenomenon is based on the formation of FCIV/FCIII ([Fe$\left.\left.(\mathrm{CN})_{6}\right]^{4-} /\left[\mathrm{Fe}(\mathrm{CN})_{6}\right]^{3-}\right)$ ion bridges between the polymer chains (Figure 10). As mentioned above, the addition of FCIV ions led to a contraction of the film. This could be explained by the formation of FCIV ion bridges between positively charged $\mathrm{PAH}$ chains replacing $\mathrm{Cl}^{-}$ions. Like that, the film contracts, and water is expelled. Simultaneously, the stronger interactions in the film make it stiffer, as can be deduced by the dissipation decrease monitored by QCM-D. The application of $600 \mathrm{mV}$ induces the oxidation of the FCIV ions. The less charged FCIII ions form weaker ion bridges, which get partially replaced by counterions, resulting in swelling of the film. The charge inside the film is compensated by the diffusion of chloride ions from the supernatant into the film. When the potential is decreased to $0 \mathrm{mV}$, FCIII ions are reduced, and at the same time, chloride ions diffuse out of the film and the FCIV-PAH bridges are reformed. Using the EC-QCM-D, we observed an increase in dissipation during the oxidation process whereas the dissipation decreased back to its original value upon reduction. This observation, as well as the associated frequency changes, suggests that the film becomes softer upon oxidation. Upon reduction, the film deswells, and its stiffness is assumed to increase.

The model proposed in Figure 10 is also able to explain our observation that the film swelling is of a higher amplitude in the absence of FCIV in solution. When the electroactive FCIV ions are present in solution, they are in competition with the chloride ions diffusing into the film during the oxidation of FCIV. One FCIV diffusing into the film will have the same effect, from an electrostatic point of view, as four chloride ions. When FCIV ions are present in solution, a lower number of anions have to diffuse into the film to maintain its electroneutrality. This will reduce the swelling effect in comparison to the case where only 
A)

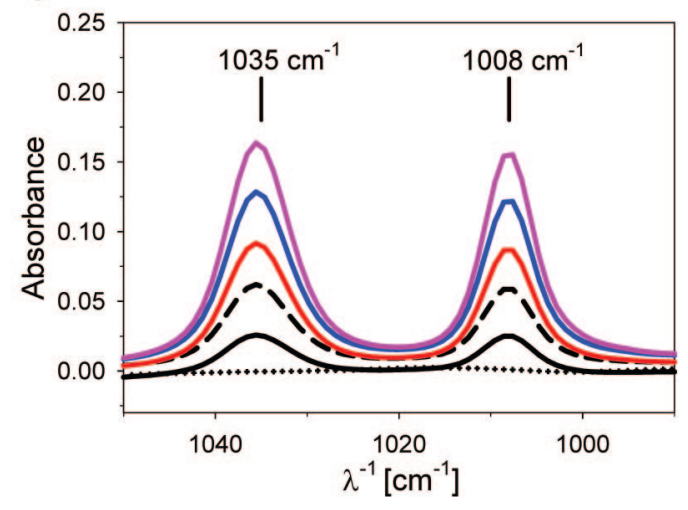

B)

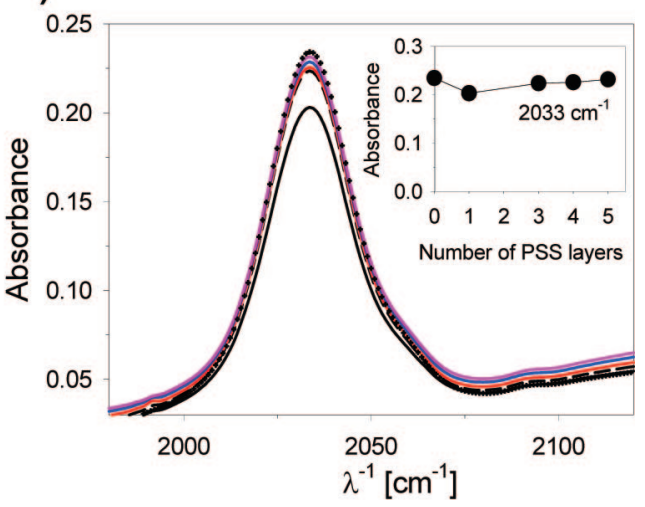

Figure 12. FTIR spectral regions characteristic (A) of the absorbance peaks of the sulfonate groups due to PSS ( $\left.980-1080 \mathrm{~cm}^{-1}\right)$ and $(\mathrm{B})$ of FCIV $\left(1950-2100 \mathrm{~cm}^{-1}\right.$ ) obtained for the PEI-(PGA-PAH) 5 film after the loading of FCIV (black dots) and after the deposition of PLL/PSS layers 1 (black line), 2 (black dashes), 3 (red line), 4 (blue line), and 5 (pink line) pairs. Because the spectra did not change upon the deposition of PLL, they have been omitted for the sake of clarity. The inset of B represents the absorbance intensity of the band at $2033 \mathrm{~cm}^{-1}$ (attributed to FCIV) upon further deposition of (PSS-PLL) layer pairs.
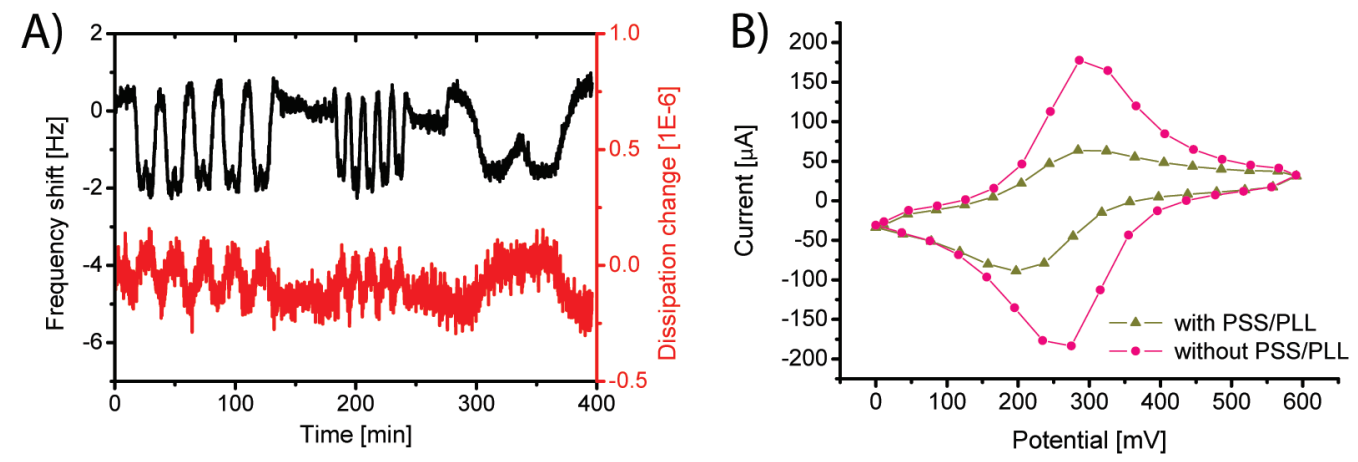

Figure 13. (A) Frequency shift and dissipation change of PEI-(PGA-PAH) $)_{5}$-FCIV-(PSS-PLL) 5 during CV at 50, 10, and 100 mV/s. (B) Voltammogram of PEI-(PGA-PAH) $)_{5}$-FCIV and PEI-(PGA-PAH) $)_{5}$-FCIV-(PSS-PLL) 5 (scan rate $100 \mathrm{mV} / \mathrm{s}$ ).

chloride ions can diffuse (absence of FCIV in solution). The swelling behavior under an electric potential related to ion uptake was also observed for polyelectrolyte-enzyme films as reported by Forzani et al. ${ }^{21}$ Compared to our system, they used Os complexes, covalently attached to a polymer backbone, as a redox-active compound and built alternating layers consisting of glucose oxidase and the $\mathrm{PAH}-\mathrm{Os}$ complexes. Their complex Os compound led to swelling behavior to a similar extent, but it was slower than in our system. The changes in thickness and refractive index during the oxidation of Os are associated with anion and water uptake and are proportional to the fraction of oxidized Os. In our case, instead of a covalent bond the redox probe is bound to the polyelectrolytes by electrostatic interactions with the same amplitude in swelling/contraction. The spontaneous entrapment seems to be easier to apply, provided that the redox probe is entrapped in the film.

Besides the PGA/PAH films, we were also interested in the behavior of PEMs consisting of different polymers. We investigated PSS/PLL films in contact with FCIV ions. CV was performed on a PEI-(PSS-PLL) 5 film during and after contact with FCIV ions in solution. With FCIV ions in solution, there was an oxidation/reduction peak (Figure 5), but it was about 10 times weaker than for PGA/PAH films. Moreover, the increased difference of the peak potential (compared to PGA/PAH) suggests that the charge transfer is diffusion-limited. Once the FCIV ions in solution were replaced by buffer, the oxidation and reduction peaks disappeared, similar to the behavior in a purely capacitive system (Figure 11). This shows that PSS/PLL films, as PSS/
PDAMAC ${ }^{34}$ and PSS/PAH films, ${ }^{35}$ did not take up FCIV ions. Moreover, EC-QCM-D measurements showed that the film did not react to an applied potential with an expansion and contraction effect. These results nicely illustrate that an uptake of FCIV ions in the film seems to be a necessary condition for obtaining swelling/deswelling behavior.

In the next step, we investigated films consisting of two different multilayers, namely, PSS/PLL and PGA/PAH, where up to five bilayers of PSS/PLL were adsorbed on top of five PGA/PAH bilayers. The film growth of PSS/PLL was found to be independent of the layers underneath, but the PSS/PLL film built on top of PEI-(PGA-PAH) 5 almost completely inhibited the swelling (only about a $2 \mathrm{~Hz}$ frequency shift and a $3 \times 10^{-7}$ dissipation change). Even though such films showed oxidation and reduction peaks in the presence of FCIV ions, after the rinsing step these peaks were smaller compared to PGA/PAH films without PSS/PLL on top. In the presence of FCIV ions in solution, FCIV ions can still diffuse toward the working electrode (the substrate) and can be oxidized during the CV. PSS/PLL on top of a PGA/PAH film retarded the diffusion of FCIV ions into the PGA/PAH film, showing a limited permeability to FCIV ions. Two PSS/PLL bilayers on top were enough to reduce the frequency change to around $30 \%$. After the rinsing step, there are fewer FCIV ions entrapped in the film that are oxidized during the application of $\mathrm{CV}$. Therefore, fewer $\mathrm{Cl}^{-}$ions diffuse into the film to maintain its electroneutrality, and the swelling/deswelling effect is smaller.

(34) Farhat, T. R.; Schlenoff, J. B. Langmuir 2001, 17, 1184.

(35) Han, S.; Lindholm-Sethson, B. Electrochim. Acta 1999, 45, 845. 
We were also interested in the effect on the swelling/deswelling of a PGA/PAH film containing FCIV ions with a PSS/PLL capping film, denoted PEI-(PGA-PAH) $)_{5}$ FCIV-(PSS-PLL) 5 . Before the application of CV on this type of buildup, the FTIR experiments allowed us to further verify that the FCIV ions remain in PGA/PAH films after the deposition of an overlaying PSS/ PLL film (Figure 12). After the uptake of FCIV into PGA/PAH films and a subsequent rinsing step, the first layer of PSS led to a small decrease in the intensity of the FCIV peak (at $2033 \mathrm{~cm}^{-1}$ ) in parallel with the increase in the intensity of PSS peaks (at 1035 and $1007 \mathrm{~cm}^{-1}$ ). During the 5 PSS/PLL bilayer depositions, the PSS peaks increased, showing presence of the terminal film. At the same time, the intensity of the FCIV peak showed only a small decrease of about $5 \%$ during the buildup of PSS/PLL bilayers (inset of Figure 12B).

The frequency shift and dissipation change during the application of CV on the PEI-(PGA-PAH) $)_{5}$-FCIV-(PSS-PLL) film, monitored by EC-QCM-D, are shown in Figure 13A. Upon the application of $\mathrm{CV}$, the frequency shift and dissipation change followed the potential change but in a dramatically reduced manner compared to those for PEI-(PGA-PAH) ${ }_{5}$-FCIV. Concerning the frequency shift (and the dissipation change), the swelling/deswelling amplitudes are respectively $2 \mathrm{~Hz}\left(3 \times 10^{-7}\right)$ and $14 \mathrm{~Hz}\left(7 \times 10^{-7}\right)$ with and without the PSS/PLL capping layer. This inhibitory effect on the swelling/deswelling of PEI(PGA-PAH) $)_{5}$-FCIV is not related to the decrease in FCIV content inside the film, as has been confirmed by FTIR (Figure 12B). In Figure 13B, the voltammogram of FCIV ions contained in a PGA/PAH film before and after the deposition of the PSS/PLL capping film shows a decrease in the Faradic current and an increase in the peak potential difference. This means that charge transfer is limited by diffusion, which could explain the inhibiting effect of the PSS/PLL capping film. This diffusion limitation induces fewer transferred electrons, with fewer $\mathrm{Cl}^{-}$ions diffusing into the film to maintain its electroneutrality thus inducing a smaller swelling/deswelling effect. During the application of $\mathrm{CV}$, the values of frequency and dissipation changes are similar for a PGA/PAH film with FCIV entrapped before or after the buildup of the PSS/PLL capping film. By plotting the number of electrons transferred during the application of CV (data not shown), we notice that in both cases (i) the swelling is proportional to the number of transferred electrons (with approximately the same slope in frequency shift, $0.5 \times 10^{-14} \mathrm{~Hz} / \mathrm{e}^{-}$) and (ii) the total number of transferred electrons is approximately the same. This means that the same number of oxidized FCIV ions induces the same amplitude of swelling for both films (i.e., PEI-(PGAPAH) ${ }_{5}$-FCIV-(PSS-PLL) 5 and PEI-(PGA-PAH) $)_{5}$ (PSS-PLL) $)_{5}^{-}$ FCIV). In summary, PSS/PLL films interfered with the diffusion of FCIV and inhibited the swelling/deswelling effect (Figure 12).

\section{Conclusions}

In this article, we have introduced a platform to reversibly change the thickness of PEM films. We have achieved a similar thickness change to that of EAPs (i.e., up to 10\%) under mild conditions and with a significantly shorter reaction time. From the tested polymer combinations, PGA/PAH films were the only ones to show the ability to swell and deswell upon application of a low potential $(<600 \mathrm{mV})$. The presented results were achieved by incorporating FCIV ions that were oxidized into the film and reduced during the applied $\mathrm{CV}$. The films reacted immediately to the oxidation and the reduction even at high scan rates of 100 $\mathrm{mV} / \mathrm{s}$. During the oxidation of the FCIV ions, the film expanded and contracted back to its original thickness during reduction. According to the results from the EC-QCM-D, the film was less rigid in its expanded state than in the contracted form. The thickness change that was found was also confirmed with an EC-AFM.

This new platform at the interface between EAPs and PEM films has the potential for various applications. It could be suitable for cell stretching because of its noncytotoxic components and the mild operating conditions, such as no drastic $\mathrm{pH}$ changes and no high voltages. Because of the flexibility of the film, a possible application could also be the active control of topology.

Acknowledgment. We thank ETH Zurich and the Germaine de Staël project for funding. Stephen Wheeler is acknowledged for his help in building the AFM cell.

Supporting Information Available: Height-mode AFM image of a PEI-(PGA-PAH) ${ }_{10}$ film built on an ITO substrate. Cross-section of the PEI-(PGA-PAH) 10 film. This material is available free of charge via the Internet at http://pubs.acs.org.

LA801875U 